\title{
Research on Effective Way to Train Rural Doctor in Xinjiang National Minority Area
}

\author{
Xinwu Zhu \\ School of Politics and Public Administration \\ of Xinjiang university \\ No.1230 Yanan Road Urumqi Xinjiang China \\ Urumqi, China \\ e-mail: zhuxinwu08@sina.com
}

\author{
Yuting Guo \\ Xinjiang Medical University \\ No.393 Xinyi Road Urumqi Xinjiang China \\ Urumqi, China \\ e-mail:531399312@qq.com
}

\begin{abstract}
Imbalanced health resources between urban and rural in national minority area, insufficient number of primary health technician, and low levels of technology of urban and rural has become a bottleneck affecting the health development of agricultural and pastoral areas in Xinjiang. This article is based on the necessity for rural doctor training in agricultural and pastoral areas in Xinjiang national minority area, and proposed an effective way to train the rural doctor.
\end{abstract}

\section{Keywords- rural health; rural doctor; training}

\section{INTRODUCTION}

In recent years, along with countries continue to increase financial investment in economic and social development of Xinjiang and policy support, the strength of the rural economy in Xinjiang significantly enhanced health and other social undertakings have also made rapid development. Xinjiang as a frontier minority area, problems such as "without sufficient medicine" in rural area, "poor for disease, poverty due to illness", the existing health resources in rural areas can not meet the demand of the farmers and herdsmen for health care still prominent. Rural doctors as core resources in rural health resources plays a very important role in the development of rural health services, the level of technology and service capabilities of the rural doctors is directly related to the quality of medical services to the grassroots farmers and herdsmen, and the right of everyone to the enjoyment of the primary health goals. The Xinjiang local colleges commitments main task, and how the culture at all levels of all types of medical and health professionals for the Xinjiang region in accordance with The CPE Central Committees and State Councils Decision on further strengthening the rural health "(The CPC central committee deemed[2002] No. 13)," the CPC Central Committee office Hall, the State Council General Office of views on further strengthening of qualified personnel in the western region (The CPC central committee general office outgoing mail [2007] No. 9) and the CPC Central Committee and State Council on strengthening the rural utility personnel and rural human resources development views Office "( The CPC central committee general office outgoing mail [2007] No.24) spirit of the document for the economic and social development in Xinjiang culture" have to go, stay, the lingua franca of the application of medical and health professionals, and continuously improve the service skills of doctors in rural areas, efforts improve the quality of rural doctors training, maintenance the Xinjiang and stability and achieve leapfrog development in Xinjiang has a very important significance.

\section{THE NECESSITY FOR RURAL DOCTORS TRAINING IN XINJING NATIONAL MINORITY AREA}

\section{A. Health Resources, the Status Quo of the Agricultural and Pastoral Areas in Xinjiang Rural Doctors \\ Training Requirements Must Accelerate}

Xinjiang is located in the northwest border of the motherland, accounting for one sixth of the country's total area, vast territory and diverse terrain, 18, states, municipalities and 88 counties (cities), 994 towns with a total population of approximately 2158.6 million [1], The rural population accounts for $62.06 \%$ of the total population, ethnic minorities, accounting for $60.42 \%$ of the population. Xinjiang economic development is relatively slow due to reasons of history, geography, natural, lagging behind, including health services, the development of social undertakings, highlight the contradiction between supply and demand of health resources, especially health resource allocation imbalances.

Various health institutions in Xinjiang,there are total 673972,800 hospital beds bit, all kinds of health professional and technical personnel and 116,000 practicing physicians per thousand population has an average of 1.79 people, hospital hospitals 3.97 beds per thousand [1]. Health institutions above the city level health technicians 7.52 million, accounting for $64.8 \%$ of the total number of people, the city has 3.14 practicing physicians, county, township following per thousand population has 0.87 practicing physicians per thousand population. County, township, village, rural three-tier health service system, all kinds of health institutions $61.21 \%$ bit accounted for $29.40 \%$ of the hospital beds, all kinds of health professional and technical personnel accounting for $32.57 \%$. Village health region 6956 , now $21.2 \%$ of the administrative villages still far below the health clinic, village health rural doctors have an average 0.89 people per thousand agricultural population with rural doctors (including hygienists) 0.59 the national average (per thousand agricultural population has 1.19 doctors in rural areas) and the western average (per thousand agricultural population has 1.01 doctors in rural areas) [1], with the Ministry of Health the rural doctors jobs requirement configuration standard requires a far cry. And 30\% of the total number of rural doctors can be seen overtook $60 \%$ of the region's population of the health workforce, basic medical services for farmers and herdsmen task, There are 
significant imbalances in the Xinjiang region distribution of health resources in urban and rural areas, insufficient number of primary health technician has become affect the bottleneck health development of the agricultural and pastoral areas of the Xinjiang region.

\section{B. Xinjiang Rural Doctors Team Status and Service Level Requirements Must Pay Attention to the Culture of the Rural Doctors}

Currently, the village doctors in rural areas of Xinjiang, is not only in the number of serious lack of existing rural doctors, there are serious problems in the educational structure, qualification, professional quality and service capabilities, According to 2007 statistics, the region's agricultural and pastoral areas accounted for only $6.3 \%$ of the total number of doctors in rural areas; rural doctors in the post, the number of rural doctors in medical education, secondary medical degree and college and above medical qualifications has all kinds of qualification, respectively, accounting for $60.47 \%$ of the total number of doctors in rural areas, $32.24 \%$ and $7.28 \%$; 20 to 39 age group accounted for $62.15 \%$ of the total number of doctors in rural areas in the post of village doctors. The rural doctors in Xinjiang status quo, vocational qualification, service level medical requirements of the new pastoral areas contradictions more obvious each day. The low level of rural doctors business, the knowledge update slow, unable to meet the needs of the community, has seriously affected the Xinjiang coordinated development of urban and rural economic and social.

\section{THE TRAINING OF DOCTORS IN RURAL AREAS OF THE XINJIANG ET H NIC MINORITY AREAS PATHWAY}

Rural doctors is a primary health technical team from the 1950s of the development, which overtake Xinjiang rural population's most basic health care, preventive health services such as health care and health promotion, and worked to improve rural health conditions, which plays a major role [2]. "The CPC Central Committee, the State Council decided on further strengthening the rural health work" (The CPC central committee deemed[2002] No. 13) to comprehensively strengthen the education of doctors in rural areas, the majority of doctors in rural areas throughout the country in 2000 with a practicing physician assistant and higher qualification ". According to this plan, Xinjiang tasks of the education and training of doctors in rural areas is arduous and urgent. To explore the realistic rural physician training methods, summarize the actual situation analysis of higher medical education in Xinjiang, it is necessary to create an entirely new mode of personnel training, give full play to the advantages of higher education resources, the use of the University various types of teaching practice base, radiation to the three medical institutions, in order to comprehensively promote the improvement of rural doctors subject knowledge, clinical skills and service levels.

\section{A. To Accelerate Job Training, and Improve the Capacity and Service Level of Existing Rural Doctors Practicing}

Services provided by rural doctors not only including health education, core services such as immunization and maternal and child health plan, but also independently health activities, should have a more comprehensive common technique to master common diagnostic and treatment technology, so the job training to "demand-oriented, focusing on actual clinical work ability, scientific expertise structure adjustment of rural doctors [3]. Combined with the actual choice by a specialist in the county-level hospitals in rotation, to promote the transformation of rural doctors separate business training to strengthen the overall quality of education, specific rotation departments by the rural physician with on-demand training, combination of study plans, system manner. But sparsely populated rural and pastoral areas of our region, over a broad area features prominent mode and form of on-the-job training to be diverse, to focus on strengthening training effect, the training content gleaning on, time flexibility, mainly to strengthen the location of doctors in rural areas and endemic diseases and vulnerable specialist knowledge, tend to rural common problem, chronic disease management, focusing on population health, community prevention.

\section{B. To Encourage Academic Education, Improve the Mechanism of Continuing Education}

The existing college educated, age, village doctors under 40 years of age, with national adult education policy, increase the intensity of training, flexible training methods, you can make use of the remote network education and learning points to impart theoretical knowledge and organize regular thematic internships to improve the practical ability to university teaching base; strengthen counseling while the completion of a higher level of education assistant practitioner qualification examination by the school organization, in order to increase its proportion of the practicing physician assistant exam, and gradually improve the rural doctors' overall level of the team. So the rural medical health employees need the corresponding government policy support in adult education, continuing education and on-the-job training, etc [4]. Health administrative department should study the effective approaches and long-term mechanism to train rural doctors, to establish the continue education mode of accord with the objective reality. The mechanism can improve rural doctors ability, can also let them "bottom go to, retain and good job", and finally rural doctors could play a bigger role in the basic unit.

\section{The Implementation of the Country's "Targeted Recruitment, Order Training" to Speed up the Training of Reserve Talents}

Based on the existing number of health personnel and pastoral areas in Xinjiang and the state of development in the next five years, in line with the basic principle to meet the health service needs of the agricultural and pastoral areas, and autonomous regions in organized high school starting point for the agricultural and pastoral areas specialist level medical education real. The opinions "developed for agricultural and pastoral areas of medical personnel orientation training plan, from 2007 to 2011, our region's existing higher medical education resources for agricultural and pastoral areas township (town), the state-run farms held in the high school starting college level medical education, directed Xinjiang township hospitals to train 4,000 specialist qualifications, practical medical expertise with the appropriate professional and technical skills, to supplement the township hospitals clinical professionals, health personnel gradually improve agricultural and pastoral areas education levels and service levels. Xinjiang Medical University, Shihezi University, Xinjiang Uygur Medical College will assume all of the training mission, which 
completed training mission will be $80 \%$ of doctors in rural areas of Xinjiang Medical University. Through the establishment of rural doctors in training mode conform Xinjiang actual need to improve the overall quality of the health of the agricultural and pastoral areas and service levels, make due contribution to the sustainable development of rural health services in Xinjiang.

\section{ACKNOWLEDGEMENT}

This research was financially supported by the Ministry of Education Social Science Foundation of China (Grant NO. 09XJA630005).

\section{REFERENCES}

[1] Ministry of Health ,2010 Health Statistical Yearbook.

[2] Li Changming, “Our country rural doctors existing historical significance and practical limitations”, Journal of Chinese Rural Physician, vo17, pp. 3〜 4, 2001.

[3] Ding Bailin, "Discuss on make full use of the resources of higher medical colleges to train rural doctors", Chinese Rural Health Service Administration, vo23, pp. 91 92, 2003.

[4] Liang Yifei,Yang Guohong, Wang jing, "Thinking about the talented person troop construction of rural medical and health”, Chinese Health Service Management, pp. 516-517, 2008.

[5] Li Jizhi, "Study on equal access to basic public servic in china under the background of the integration of urban and rural areas", Agricultural Modernization Research, Vol. 33 No.4,pp. 451-455, 2012 